\title{
Increased dose primary thromboprophylaxis in ambulatory patients with advanced pancreatic ductal adenocarcinoma, a single centre cohort study
}

Anthony Maraveyas ${ }^{1,2^{*}}$ (D) Farzana Haque ${ }^{1,2}$, lqtedar Ahmed Muazzam ${ }^{1}$, Waqas llyas $^{1}$ and George Bozas ${ }^{1}$

\begin{abstract}
Background: Advanced pancreatic ductal adenocarcinoma (aPDAC) patients have a lifetime all type thromboembolic event (ATTE) rate of 25-35\%. Efficacy and safety of increased dose primary thromboprophylaxis (IDPTP) with low molecular heparin (LMWH) given for 3 months has been shown in two prospective randomized trials.
\end{abstract}

Objectives: To report on efficacy -reduction of all type thromboembolic events (ATTE)-, safety -incidence of Major Bleeding (MB)- and compliance in a single-centre cohort of aPDAC patients receiving first line chemotherapy and LMWH-IDPTP.

Methods: From May 2009 to October 2016, 82 patients received IDPTP -LMWH with dalteparin. Schedule: 55 kg and below: $7500 \mathrm{IU}$, between 55 and $80 \mathrm{~kg}: 10,000 \mathrm{IU}$, above $80 \mathrm{~kg}: 12,500 \mathrm{IU}$. MB is reported using the International Society of Thrombosis and Haemostasis (ISTH) criteria. ATTE was defined as any arterial or venous event, incidental or clinically symptomatic, including visceral VTE.

Results: Mean and median time on dalteparin was $10.2(95 \% \mathrm{Cl} 8.1,12.4)$ and $8.0(95 \% \mathrm{Cl} 6.2,9.7)$ months respectively. ATTE was observed in 7 (8.5\%) of patients, with a median time on IDPTP of 6.2 months (95\% Cl 10.0, 13.2). MB was seen in 10 (12.2\%) patients with a median time on IDPTP of 4.5 months ( $95 \% \mathrm{Cl}$ 1.6, 7.4). Six major bleeds (60\%) were the direct or indirect result of aPDAC. Eighty-one patients had died at the time of data collection with a median overall survival time of 8.7 months $(95 \% \mathrm{Cl} 6.4,11.0)$. Thromboembolism and bleeding were late events. No impact of thromboembolism or bleeding on overall survival was observed.

\footnotetext{
* Correspondence: anthony.maraveyas@hey.nhs.uk

'Queen's Centre for Oncology and Haematology, Castle Hill Hospital, Hull University Teachng Hospitals (HUTH), Cottingham HU16 5JQ, UK ${ }^{2}$ Joint Centre for Cancer Studies, Faculty of Health Sciences, The Hull York Medical School, University of Hull, Cottingham HU6 7RX, UK
}

C C The Author(s). 2020 Open Access This article is licensed under a Creative Commons Attribution 4.0 International License, which permits use, sharing, adaptation, distribution and reproduction in any medium or format, as long as you give appropriate credit to the original author(s) and the source, provide a link to the Creative Commons licence, and indicate if changes were made. The images or other third party material in this article are included in the article's Creative Commons. licence, unless indicated otherwise in a credit line to the material. If material is not included in the article's Creative Commons licence and your intended use is not permitted by statutory regulation or exceeds the permitted use, you will need to obtain permission directly from the copyright holder. To view a copy of this licence, visit http://creativecommons.org/licenses/by/4.0/ The Creative Commons Public Domain Dedication waiver (http://creativecommons.org/publicdomain/zero/1.0/) applies to the data made available in this article, unless otherwise stated in a credit line to the data. 
(Continued from previous page)

Conclusions: IDPTP-dalteparin was associated with lower ATTE occurrence rates than expected and comparable major bleeding rates. ATTE and MB were late events, the majority of MB was from direct or indirect result of locally progressing aPDAC. Since these conditions can frequently arise in aPDAC, IDPTP should be regularly reviewed beyond 3 months.

Keywords: Pancreatic cancer, Thromboprophylaxis, Dalteparin, Ambulatory, Chemotherapy

\section{Introduction}

Patients with advanced pancreatic ductal adenocarcinoma (aPDAC) have a seven-fold increased risk of developing arterial and venous thromboembolism as compared to patients with most other solid and haematological malignancies $[1,2]$. This translates to about one-third of patients with aPDAC suffering from either a clinically evident or unsuspected (incidental) thrombo-embolic event (TE) over short life spans, and the spectrum of these events is broad, ranging from the conventional venous thromboembolism (VTE) of deep vein thrombosis (DVT)-pulmonary embolism (PE) complex, to arterial as well as visceral thromboses [3]. The manifestation of a VTE, apart from the physical and psychological burden inflicted on the cancer patient, generally results in increased health utilization costs of up to $45 \%$ per affected cancer patient [4] with VTE incremental costs being the highest in pancreatic cancer patients [5].

The administration of chemotherapy in ambulatory aPDAC patients is an additional risk factor for thromboembolism which further complicates an already challenging management [3]. A modest improvement in survival has been seen with new chemotherapy combinations [6] but they have also resulted in increased VTE risk [7]. The use of currently available anticoagulant agents reduces VTE risk and their efficacy has been proven in randomized trials $[8,9]$. Current guidelines from major international bodies have changed recently to suggest that patients with Khorana Score 2 and above should be considered for thromboprophylaxis. This by definition includes patients with pancreatic cancer [10-12]. These guidelines have been updated following the studies AVERT $[13,14]$ and CASSINI $[14,13]$ and now include the option of using a direct oral anticoagulant (DOAC). The recent (2018) NICE guidance also suggests that thromboprophylaxis should be considered in aPDAC [13, 15]. Both the FRAGEM study [8] with a $19.6 \%$ absolute difference in thromboembolic rates and a number needed to treat (NNT) of 5 and CONKO-04 [9] with an absolute difference of $8.7 \%$ in thromboembolic rate and a NNT of 11 provide meaningful clinical benefit.

These studies used increased dose primary thromboprophylaxis (IDPTP) for 3 months. The investigators in CONKO 004 used a two-third -therapeutic dose of enoxaparin ( $1 \mathrm{mg} / \mathrm{kg}$ once daily) for the first 3 months, followed by conventional primary prophylaxis maintenance of $40 \mathrm{mg}$ sc. once daily. The investigators in FRAGEM used full therapeutic dose schedule of dalteparin $(200 \mathrm{u} / \mathrm{kg}$ once daily for 4 weeks reduced to $150 \mathrm{u} / \mathrm{kg}$ once daily) for the first 3 months at which point thromboprophylaxis was discontinued.

None of the current guidelines offer a clear opinion on LMWH dosing though the ASCO guideline mentions that the studies of LMWH thromboprophylaxis in pancreatic cancer patients were conducted at a 'treatment dose' of LMWH [10] otherwise termed 'supra-prophylactic' [16].

Soon after the LMWH-related data were first published Hull University Teaching Hospitals (HUTH) implemented a dalteparin-based thromboprophyalxis schedule for aPDAC patients using a supra-prophylactic dosing approach. It is this single-centre retrospective real-world cohort series experience in ambulatory aPDAC patients that we present here.

\section{Objectives}

To report efficacy, safety and compliance in a real-world cohort of consecutively treated patients with advanced and metastatic pancreatic cancer receiving an increased dose primary thromboprophylaxis (IDPTP) schedule of dalteparin.

\section{Patients and methods Increased dose primary thromboprophylaxis (IDPTP) schedule}

For aPDAC patients deemed fit for systemic treatment we implement a simplified dalteparin increased dose thromboprophylaxis schedule, close to the $75 \%$ full therapeutic dose of the CLOT schedule used in the FRAGEM trial and in accordance with the CONKO-04 scheduling of continuing thromboprophylaxis throughout chemotherapy but without mandating a switch to conventional dose or discontinuation.

The IDPTP dalteparin schedule was weight adjusted and ranged as follows. For patients $55 \mathrm{~kg}$ and below: $7500 \mathrm{IU}$, for patients between 55 and $80 \mathrm{~kg}: 10,000 \mathrm{IU}$ and for patients above $80 \mathrm{~kg}: 12,500 \mathrm{IU}$.

\section{Patients}

The HPB-MDT database (Taunton \& Somerset NHSFT QGCE 1499) was searched for all pancreatic cancer 
patients that were referred to the cancer centre between May 2009 and October 2016, and then cross referenced with the electronic chemotherapy database (ARIA MedOnc 13.7). Patients were further cross referenced through the electronic medical record system (iSOFT Patient Centre ${ }^{\circ}, \mathrm{CSC}^{\mathrm{mm}}$ and Lorenzo $\left.{ }^{\circ}, \mathrm{CSC}^{\mathrm{rm}}\right)$ and through the pharmacy dispensary database (Ascribe $\mathrm{RX}^{\mathrm{Tm}}$ ) for all the outpatient prescriptions for anticoagulants. From these electronic medical records demographic, historic, cancer therapy and cancer specific data were collected for each patient in a retrospective manner. All patients had histological/cytological proof of ductal adenocarcinoma of the pancreas with inoperable locally advanced or metastatic cancer and an multi-disciplinary team (MDT) decision for systemic treatment. Only patients due to receive first line treatment were included. Accordingly, patients had to have haematological and biochemical indices allowing systemic treatment for their cancer to be eligible for treatment with IDPTP. Non-ambulatory patients or patients not considered for systemic cancer treatment either due to performance status or non -alleviated cancer morbidity (e.g. non-relievable obstruction of biliary tree or duodenal outlet) were excluded. Patients with any contraindications for anticoagulation (dalteparin) were excluded. Patients receiving other anticoagulation (e.g. a direct oral anticoagulant or warfarin) and not transferred to IDPTP were excluded.

\section{Outcome measures}

Primary aim of the study was to determine the occurrence rate of all type thromboembolic events (ATTE) and rate of major bleeding (MB). ATTE was defined as any TE event -arterial or venous, incidental or clinically symptomatic including visceral VTE and is reported in this manuscript as ATTE or TE. VTE is used for conventional PE/DVT definitions. All recorded ATTE and bleeding events were reviewed by three of the authors (AM, GB \& IM) and the nature, severity and classification agreed. Bleeding episodes were classified into major and minor by ISTH criteria. Major bleeding was defined as any fatal bleeding, symptomatic bleeding in a critical area or organ, such as intracranial, intraspinal, intraocular, retroperitoneal, intra-articular or pericardial, or intramuscular with compartment syndrome, and/or bleeding causing a fall in haemoglobin level of $20 \mathrm{~g} \mathrm{~L}^{-1}$ $\left(1.24 \mathrm{mmol} \mathrm{L}^{-1}\right)$ or more, or leading to transfusion of two or more units of whole blood or red cells [17]. Compliance to anticoagulant treatment was inferred from the time and length of the last recorded outpatient or ward prescription chart.

\section{Approvals \& Statistics}

The study reported in this manuscript is the result of work that has been classified as an audit. This is a regular undertaking in UK hospitals with the primary goal of maintaining quality standards and identifying areas that need improvement if they fall below accepted standards as set by national or international guidelines. Audits can be retrospective or prospective. As per the NHS Health Research Authority guidelines our study, which can be classified within the audit / service evaluation description, does not require external Research Ethics Committee approval [18]. The NHS Trust governance body which authorises the project is doing so if the study is conducted within the regulatory framework including the Data Protection Act (1998), the Caldicott principles (1997) and the NHS Confidentiality code of practice (2003) [19]. Within this context regulatory approval is sought and obtained based on the quality of the audit and the priority of the area studied and whether it fits within the quality framework of the organization. The endorsement code for our study is 2017.98 issued by the Hull and East Yorkshire Hospitals NHS Trust approved the 1st June 2017.

Patients were registered sequentially on the database. Data were collected in an MS EXCEL 2010 (Microsoft Corp $\left.^{\mathrm{Tm}}\right)$ spread sheet maintained in a secure virtual harddrive with restricted access. All analyses were performed with SPSS ver22, IBM Corp ${ }^{\circ}$. Descriptive statistics were used to analyse patient characteristics. Data on the TEfree interval (TFI) and bleeding-free interval (BFI) were reported in months from the time of commencing IDPTP up to the index event (last follow-up contact or the date of death). The collected data was also used to calculate the median overall survival of the whole study population and whether TE and bleeding episodes had demonstrated any impact on survival. No patients were lost to follow-up. To correct for competing risk of death the rate of TE or major bleeding for the time intervals was calculated as the number of events divided by the total number of patients at risk (subject months). The Clopper-Pearson method was used for 95\% confidence interval. The Kaplan Meier method was utilised to explore the prognostic significance of categorical variables using the log rank test to compare factors.

\section{Results}

One hundred and one patients with aPDAC were registered on the database. The search process also identified patients with prior VTE either before cancer diagnosis or during adjuvant treatment for PDAC, patients with concurrent diagnosis of $\mathrm{TE}$ and aPDAC or patients on long term anticoagulants due to pre-existing AF (atrial Fibrillation) or unprovoked VTE having been switched to an IDPTP schedule (details in Flow chart Fig. 1).

Demographics and event rates are reported for the whole cohort but the detailed analyses are restricted to 


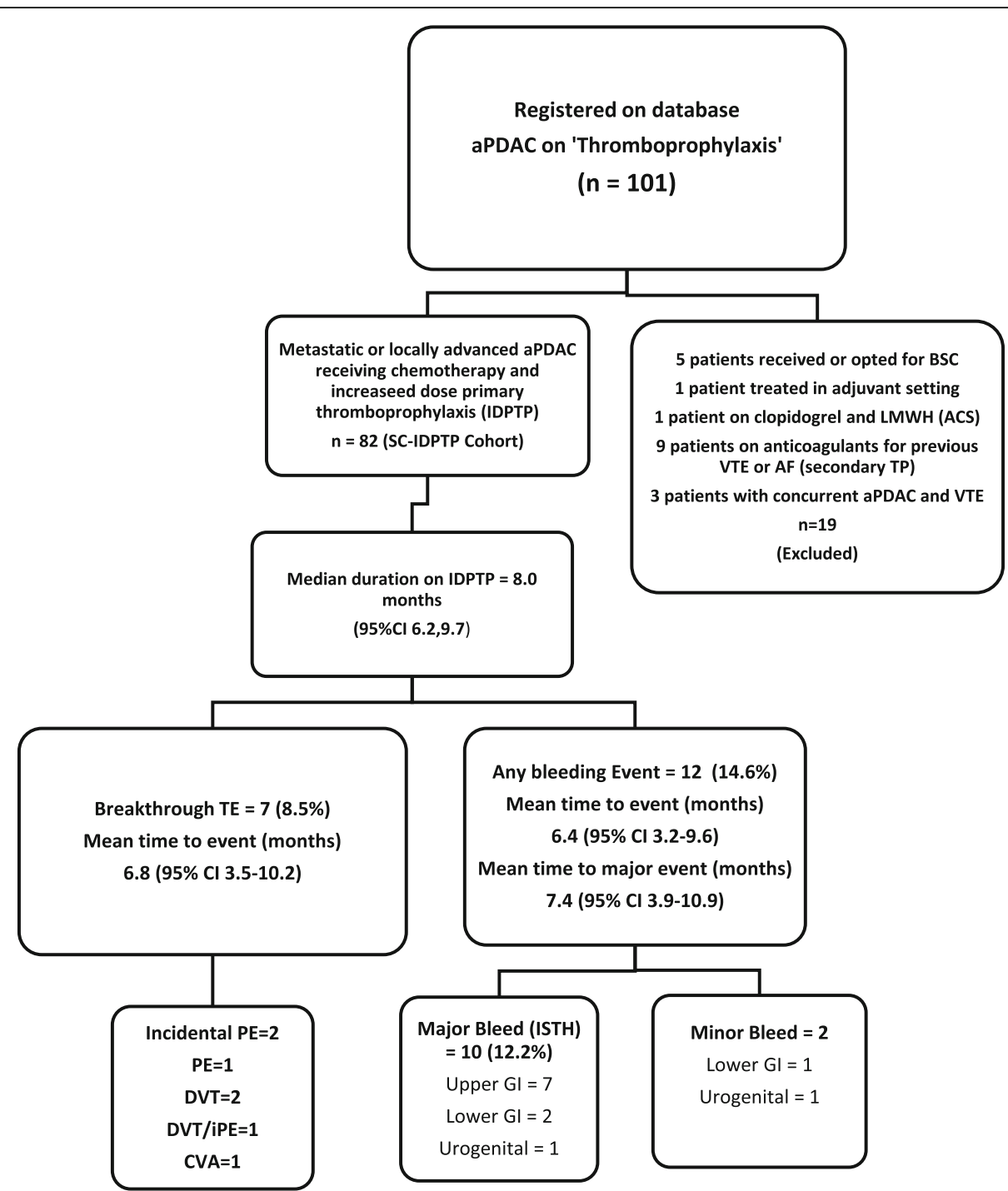

Fig. 1 Study Flow Chart. Patients that were identified using the search strategy described in the methods were entered on to a data base. Their case notes were reviewed and the patients that bore similarities to the experimental cohort of patients treated in the FRAGEM [8] and CONKO-04 [9] studies were selected for further analyses (IDPTP group). The miscellaneous reasons for exclusion are detailed. (ACS = Acute Coronary Syndrome, AF = Atrial Fibrillation, aPDAC = advanced Pancreatic Ductal Adenocarcinoma, BSC = Best Supportive Care, CVA =Cerebrovascular Accident, DVT = Deep Vein Thrombosis, GI = Gastrointestinal, iPE = Incidental Pulmonary Embolism, LMWH = Low Molecular Weight Heparin, $\mathrm{PE}=$ Pulmonary Embolism, SC-IDPTP $=$ Study Cohort of Increased Dose Primary Thromboprophylaxis, TE = Thromboembolic Event, TP = Thromboprophylaxis, VTE = Venous Thromboembolism)

the 82 patients that corresponded to an entirely IDPTP cohort.

The median age was 67 years, interquartile range: $61-72$ $(n=82)$ and $54 \%$ were male. Sixty five per cent of these patients were metastatic and the rest locally advanced. Ninety-six patients were offered first line chemotherapy. Sixty nine per cent of the patients received only one line of treatment, $20 \%$ received two lines of treatment and only $2 \%$ received a third line of treatment.

Of the IDPTP cohort $(N=82) 64$ patients $(78 \%)$ were offered Gemcitabine based first line treatment (48 patients (58\%) single agent gemcitabine \& 16 (20\%) first line gemcitabine-based combination) and 18 patients FOLFIRINOX.

The baseline characteristics of all patients, for the 82 IDPTP patients and the primary endpoint data are shown in Table 1.

In the IDPTP cohort $(n=82)$, patients remained on thromboprophylaxis for a median duration of 8.0 months,95\% CI $(6.2,9.7)$. Seven patients $[8.5,95 \% \mathrm{CI}$ $(3.7,14.6)]$ developed a TE despite IDPTP and ten patients $[12.2,95 \%$ CI $(6.1,19.5)]$ in the IDPTP group had a major bleed and 2 patients [2.4 95\%CI $(.0,6.1)]$ had a minor bleed, for a combined event rate of major 
Table 1 Patient Characteristics and Events (Major and Minor Bleeding \& All-type Thromboembolic Events). Data shown for both the IDPTP Cohort and for thepatients that were excluded

\begin{tabular}{|c|c|c|}
\hline & Miscellaneous TP & IDPTP \\
\hline Patients & 19 & 82 \\
\hline Age (years) & $71(r 41)$ & $67(r 51)$ \\
\hline Male & $11(58 \%)$ & $43(52 \%)$ \\
\hline Metastatic & 10 (53\%) & $56(68 \%)$ \\
\hline WHO PS 0-1 & 10 (53\%) & 67 (82\%) \\
\hline Chemotherapy & ${ }^{\mathrm{a}} 14(73 \%)$ & b82 (100\%) \\
\hline FOLFIRINOX & $2(10 \%)$ & $18(22 \%)$ \\
\hline Gemcitabine SA & $10(52 \%)$ & 48 (58\%) \\
\hline Gem-Based Combi & $1(5 \%)$ & $16(20 \%)$ \\
\hline No Chemo & $5(27 \%)$ & - \\
\hline Events (Bleeding) & $4(20 \%)$ & $12(14.5 \%)$ \\
\hline \#Major Bleeding & $2(10 \%)$ & $10(12.2 \%)$ \\
\hline Upper Gl bleed & $2(100 \%)$ & $7(70 \%)$ \\
\hline Lower Gl bleed & - & $2(20 \%)$ \\
\hline Genitourinary & & $1(10 \%)$ \\
\hline \#Minor Bleeding & $2(10 \%)$ & ${ }^{c} 2(2.5 \%)$ \\
\hline Events (AT-TE) & $7(37 \%)$ & $7(8.5 \%)$ \\
\hline DVT & 2 & 2 \\
\hline $\mathrm{i}-\mathrm{PE}$ & 2 & 2 \\
\hline PE & - & 1 \\
\hline i-PE \& DVT & - & 1 \\
\hline CVA & 2 & 1 \\
\hline DVT \& Visceral & 1 & \\
\hline
\end{tabular}

ane patient was treated with adjuvant chemotherapy (5FU/LV)

${ }^{\mathrm{b}} 73 \%$ completed one line, $24 \%$ two lines \& $2.4 \%$ three lines of chemotherapy

${ }^{\mathrm{c}} 1$ minor bleed was upper $\mathrm{Gl}$

bleeding and TE of $18 \%$ [ $n=15 / 82$ as two patients had a TE and a bleed, 95\%CI $(9.8,26.80)]$. The correlation between the IDPTP and the timing of TE and bleeding can be seen in Fig. 2. The cumulative rates of MB at 3 and 12 months of this real-world cohort are compared to the experimental arms of FRAGEM and CONKO-04 where data were available (Table 2). Figure $2 \mathrm{a} \& \mathrm{~b}$ present the cumulative hazard of developing a breakthrough TE or bleed. The accelerated phase of the cumulative hazards plot is between 4 and 8 months which is later than the conventional first 3 months seen in patients without thromboprophylaxis, reflecting the median time to TE of 6.2 months (95\% CI 10.0, 13.2). This can be seen most clearly in Fig. 2c where the data are corrected for competing risk of death. A similar late pattern is noted for the major bleeding episodes median time to $\mathrm{MB} 4.5$ months (95\% CI 1.6, 7.4). The majority of the minor and major bleeding events were upper GI $(8 / 14,57 \%)$ and were directly or indirectly attributable to an aPDAC related cause.
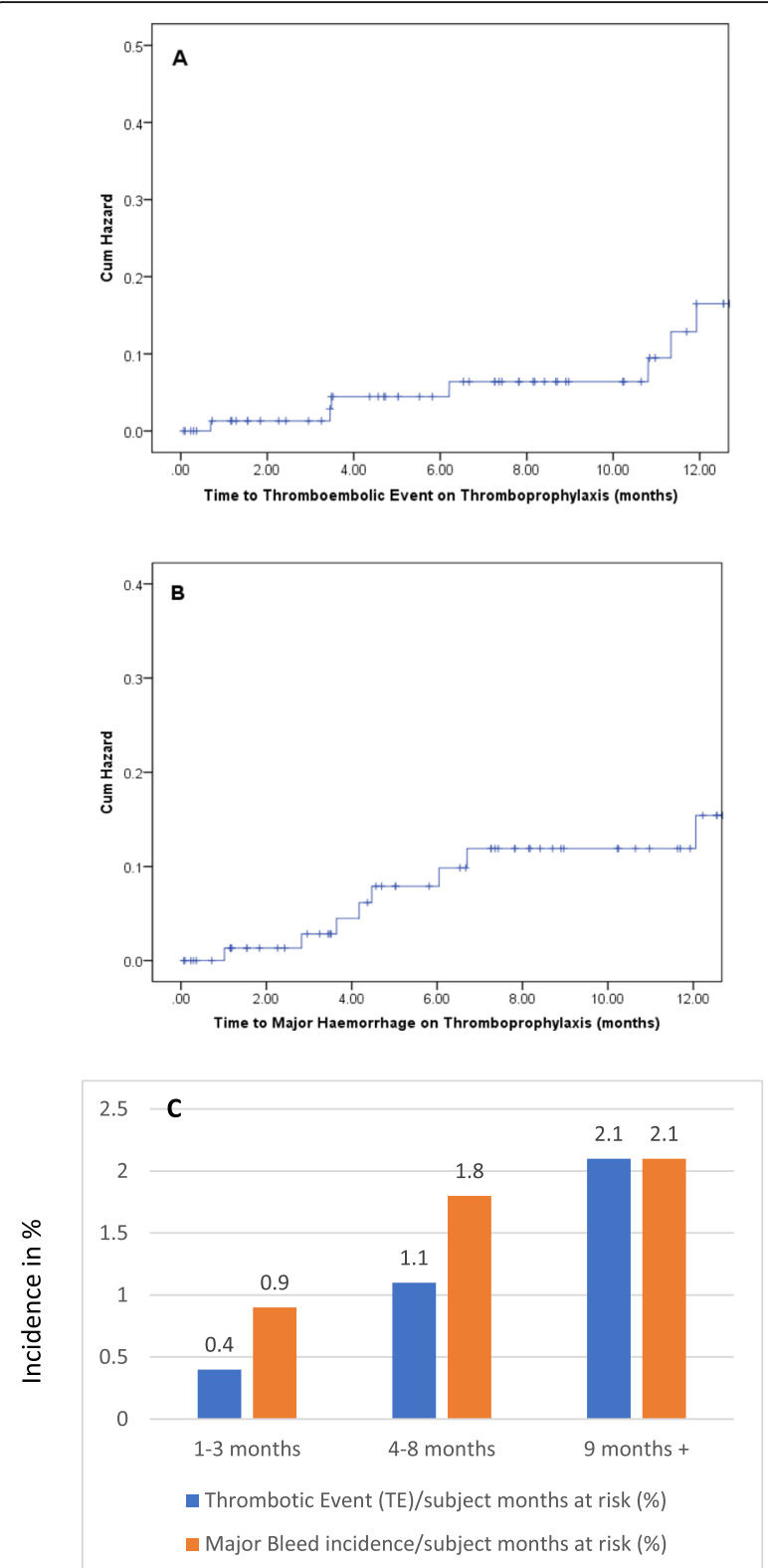

Fig. 2 The Hazard Plots (Kaplan Meier) for: a TE events, (b) Major Haemorrhage for the first 12 months of follow-up of 82 patients on IDPTP. c Incidence of thrombembolic events (TE) and Major Bleeding at different time periods. A lower incidence of TE is seen during the 1st 3 months, $0.4 \%$ ( $95 \% \mathrm{Cl} 0.0,2.4 ; 1 / 226$ subject months), compared to $2.1 \%$ ( $95 \% \mathrm{Cl} 0.4,5.9 ; 3 / 146$ subject months) after 8 months (the median duration of thromboprophylaxis in this group). A similar pattern is notable for major bleeding. Incidence was less in the 1st 3 months, $0.9 \%$ ( $95 \% \mathrm{Cl} 0.1,3.1 ; 2 / 227$ subject months). During the $4-8$ months period (280 subject months), 5 patients experienced a major bleeding event that gives an incidence of $1.8 \%(95 \% \mathrm{Cl} 0.6,4.1)$. Comparable to that, 3 patients (146 subject months) had major bleed after 8 months with an incidence of $2.1 \%(95 \% \mathrm{Cl} 0.4,5.9)$

Median overall survival time from commencement of IDPTP was 8.7 months, 95\%CI, $(6.4,10.9)$. Neither thrombotic events [12.2 months, 95\% CI $(10.7,13.7)$ 
Table 2 Cross-study comparison of three- and 12 month landmark events (Major Bleeding and Thromboembolism)

\begin{tabular}{llll}
\hline EVENTS (Cumulative Incidence) & CONKO-04 & FRAGEM & SC-IDPTP \\
\hline Major Bleed (3 Months) & $4.5 \%(7 / 160)$ & $3 \%(3 / 59)$ & 2.4\% (2/82) \\
Major Bleed (12 months) & $8.3 \%(13 / 160)$ & Not Reported (N/R) & 9.7\% (8/82) \\
Breakthrough PE/DVT (12 months) & $5.7 \%(9 / 160)$ & ${ }^{\mathrm{a}} 8.4 \%(5 / 59)$ & $\mathrm{b} 4.8 \%(4 / 82)$ \\
Breakthrough All type TE (12 months) & Not Reported (N/R) & $12 \%(7 / 59)$ & $8.5 \%(7 / 82)$ \\
\hline
\end{tabular}

${ }^{a}$ Excludes 2 cases of I-PE to be comparable to CONKO-04

${ }^{\mathrm{b}}$ Excludes 2 cases of I-PE and the CVA so as to be comparable to CONKO-04

Vs 8.4 months, 95\% CI $(6.9,9.9)(p=0.79)]$ nor bleeding events [median 8.7 months, 95\% CI $(0.4,17)$ Vs 8.4 months, $95 \%$ CI $(5.8,11)(p=0.94)]$ affected survival outcome. Progressive cancer remained the major cause of death (70 out of $82 ; 85 \%$ ) as reported in the death certificate and agreed by the investigators. Five of these patients were found to be suffering from $\mathrm{MB}$ as a tumour progression-related event during the process of dying from cancer, the context often informing decision making (e.g. restraint on endoscopic or other invasive procedures or reflection on futility of transfusion). For one patient bleeding (uterine) was reported in the death certificate and confirmed by the investigators as the direct cause of death (1.2\%). Two patients suffered a fatal thromboembolic event (2.4\%). One a CVA and one a pulmonary infarct. Figure 3 charts MB and ATTE in the relevant patients, their timing and their link to the

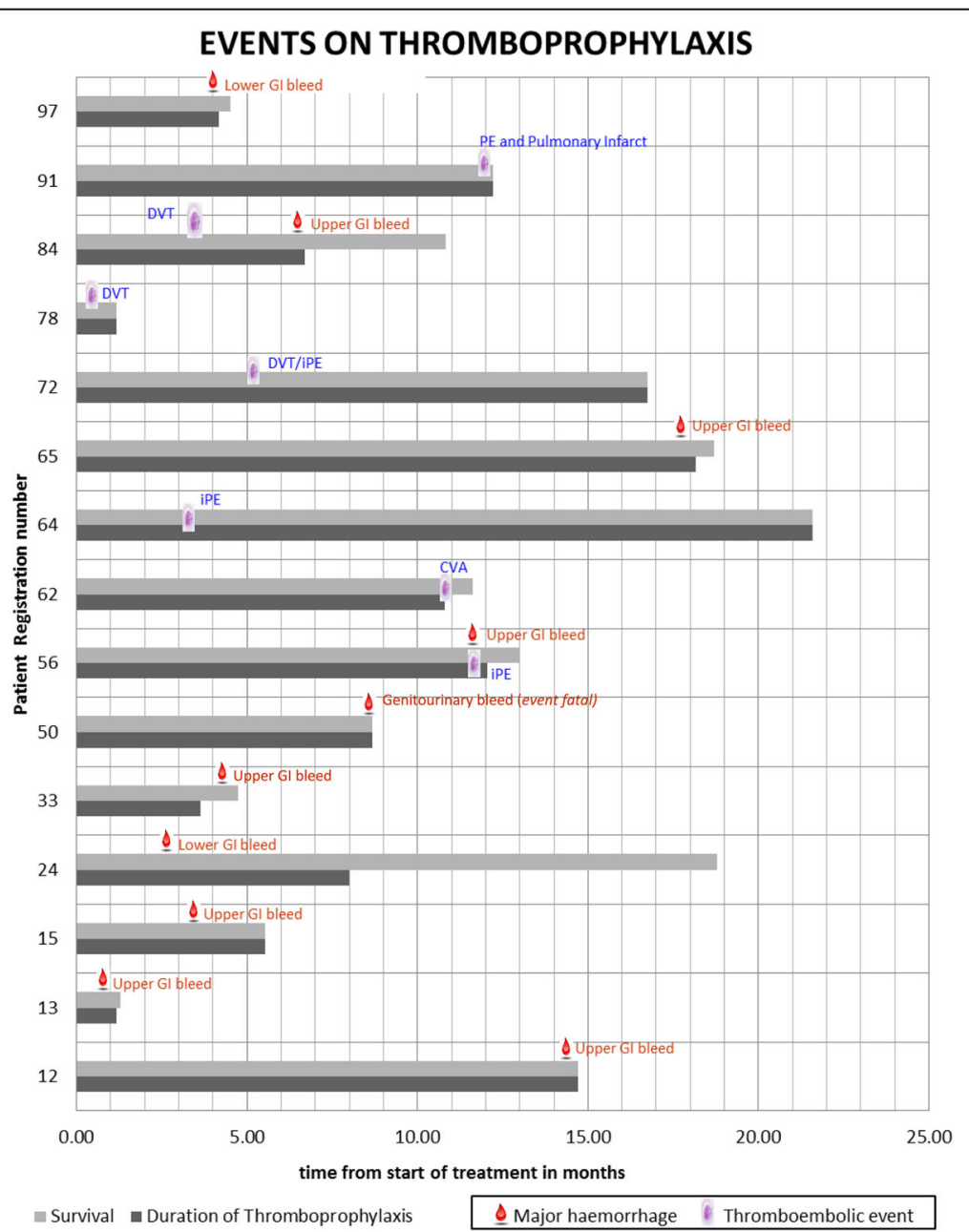

Fig. 3 The 15 patients with events. The sequential database numbers have been retained. (Gl = Gastrointestinal, iPE = Incidental Pulmonary Embolism) 
presence or absence of IDPTP over the patient lifespan.

\section{Discussion}

In this study we show that the 12 month rate of $\mathrm{TE}$ $(8.5 \%)$ is on a par with the TE rate seen in the experimental arm of the FRAGEM study (12\%) and much lower than the control arm of FRAGEM (24\%). It is also much lower than in historical series (25-35\%) [20]. We also show an incidence of clinically apparent VTE rate (PE and DVT) is 4.8\%, similar to CONKO-04 (5.7\%) and lower than in FRAGEM (8.4\%) (Table 2).

The existing RCT evidence pertains to patients receiving first line chemotherapy Gemcitabine either as a single agent or in combination with a platinum $[8,9]$. In this study $42 \%$ of patients received the more modern combinations of FOLFIRINOX (5-Fluorouracil, Irinotecan, Oxaliplatin) or AG (Abraxane, Gemcitabine). These chemotherapy schedules do not seem to have a diminished thrombosis risk. FOLFIRINOX has a $60 \%$ increase in risk of VTE (6.6\% compared to gemcitabine alone 4.1\%) [21]. In the study HALO-109-202 the rate of VTE in the investigational arm (AG-PEGPH20) was $42 \%$ and control arm (Gem Abraxane) was 25\% respectively in stage 1 of the trial [22]. $40 \mathrm{mg}$ of enoxaparin saw no VTE reduction in either arm. Only when an enoxaparin-IDPTP schedule was implemented did VTE rate drop to 5 and $6 \%$ respectively. A LMWH scale of dose-effectiveness in pancreatic cancer patients has recently been reported and can aid in decision making on IDPTP [7].

Of cancer patients commencing chemotherapy and who experience a VTE, 18.1\% will have their first event within the first month, $47 \%$ within the first 3 months, and $72.5 \%$ within the first 6 months after starting chemotherapy [23].

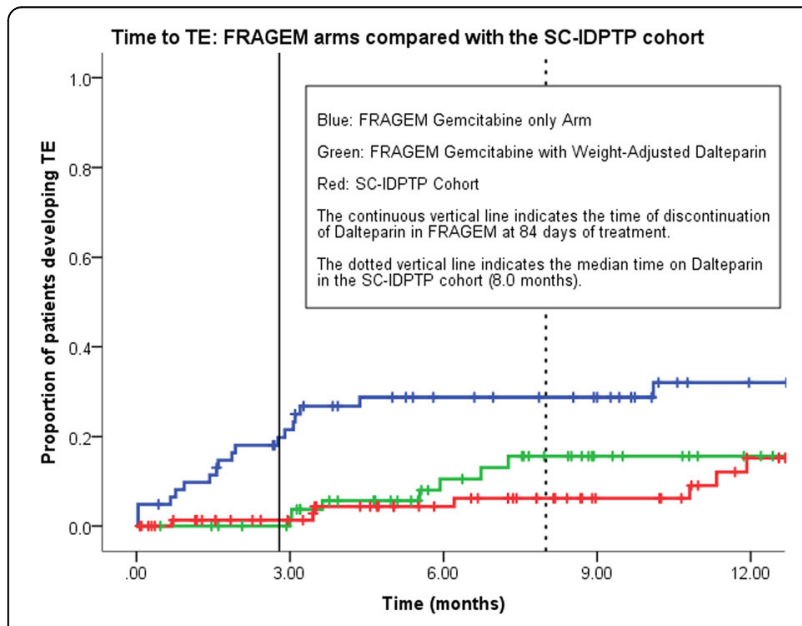

Fig. 4 Cumulative Thromboembolic Events (TEs) graph comparing TE occurrence in the two FRAGEM study arms [8] to the TE occurrence in the SC IDPTP. (SC-IDPTP = Study Cohort of Increased Dose Primary Thromboprophylaxis, TE $=$ Thromboembolic Event)
In our cohort mean time to thrombosis was 6.8 months and TE-rates continue to remain diminished over the first 10 months. This is longer than seen in the FRAGEMLMWH arm (Fig. 4). The mandatory stop of thomboprophylaxis at 84 days in FRAGEM is likely to have influenced these findings, whereas in this cohort median time on IDPTP was 8.0 months.

Figure 2a demonstrates a very late (around 12 months) manifestation of increasing TE events and may reflect the difficulties and ambivalence of maintaining thromboprophylaxis in patients arriving at the pre-terminal/terminal phase of malignancy. Adherence to anticoagulants may at that point be challenging or deemed futile. Of the 82 patients we report on, 81 had died at the time of data collection. Given the retrospective nature of the data collection for many patients we were not able to establish exact date or cause of LMWH discontinuation.

In this cohort we show that the 12 month MB rate was $12 \%(10 / 82)$. Sixty percent $(6 / 10)$ of these related to direct (infiltration) or indirect (varices) effects of the malignancy. Of the four remaining major bleeds benign pathology was documented in 2 [uterus (fibroids) and anus (haemorrhoids)] while one upper and one lower GI bleed remained unattributed. Major bleed was an end of life feature in 5 patients but death attributable solely to bleeding was documented in only one case, while in 4 it was part of the end-of-life (EoL) process of pancreatic cancer progression being the underlying cause of death and the management of the bleed tailored to the end of life healthcare context (e.g. restraint on endoscopic or other invasive procedures). There was no survival difference between patients with either TE or bleeding compared to no events.

These data are certainly in keeping with real world data for patients with aPDAC not on thromboprophylaxis [24]. Wang et al. reported on 246 cases of aPDAC, and found an overall bleeding incidence of 13\% (32 patients). A multivariate analysis showed that only stage IV disease maintained predictive significance for a bleed [HR 21.94, 95\% CI (2.274-211.642)]. Haemorrhage was an end of life event in 7\% (18 patients out of 246) [24] with a median time to death of $31+/-21$ days. No impact of bleeding on survival was seen [HR $1.246,95 \% \mathrm{CI}$ (0.754-2.060)] [8.7 months,95\% CI $(0.4,17)$ Vs 8.4 months, $95 \%$ CI $(5.8,11)]$.

Compliance has been shown to be better with oral agents with large early discontinuation rates for LMWHs [25]. Although injectable anticoagulants are undoubtedly more invasive than a tablet, patient views are nuanced. At least 2 studies $[26,27]$ have shown that patients will select anticoagulants that are least likely to interfere with their cancer treatment. In the SELECT-D sub-study [27] patients did express a preference for an oral agent, but only if this has equivalent clinical benefit to LMWH. Compliance in this 
study was very high, with patients staying on dalteparin for a median of 8.0 months. This may also reflect the absence of an alternative oral option in the IDPTP setting.

Very recently an experience of therapeutic dose rivaroxaban ( $15 \mathrm{mg}$ po bd followed by $20 \mathrm{mg}$ po od) in $28 \mathrm{aPDAC}$ patients receiving Gemcitabine Abraxane showed a 7\% major bleeding (2/28) and one breakthrough VTE (asymptomatic) $3.5 \%$ [28]. Though promising there are no randomised data in this setting for supraprophylactic DOAC schedules and one has to be aware of the greater propensity of the GI tract cancers to be associated with increased non-major but clinically relevant bleeding as seen in the recent studies of cancer associated thrombosis treatment with rivaroxaban and edoxaban $[29,30]$.

Two RCTs using conventional thromboprevention schedules of the direct oral anticoagulants rivaroxaban and apixaban in cancer patients have recently published $[13,14]$.

Patients were risk stratified using the Khorana risk stratification score. In the CASSINI study $54 \%$ of patients had either aPDAC (33\%) or Gastroesophageal (GO) (21\%) while in the AVERT study only $16 \%$ belonged to this group (13\% aPDAC and 3\% GO). Efficacy endpoint subanalysis (prevention of VTE) for the aPDAC patients has been published only for the CASSINI study (HR 0.70, 95\%CI 0.344,1.432). The latest iteration of most guidelines (2019) reinforced by the 2 recent randomised studies of DOACS, have formulated thromboprophylaxis recommendations for aPDAC [10, 11]. As the data stand LMWH-IDPTP schedules remain a proven approach for aPDAC while for the DOACs there remains the need for a well-designed single cancer RCT [31].

Our study has shortcomings; the obvious one being that this is a retrospective series and some endpoint comparisons are made to historical data. A further weakness is that we cannot account for selection bias, as we cannot identify and report in retrospect on outcomes of patients that were not offered IDPTP or not switched to IDPTP either due to contraindication or due to omission. Whilst the chemotherapy regimens were mostly gemcitabine based they included patients on different trials with experimental combinations and at least $18 \%$ on triple combination FOLFIRINOX. Nevertheless over this period, and contrary to many other cancers, the overall treatment of pancreatic cancer has brought little material change to outcomes. The study is too small to pick up substantial signals of mortality due to adverse events. Our median survivorship for the group is similar with that expected for aPDAC patients as seen in other series and studies. Although the IDPTP-dalteparin guideline adhered to in this study concurs with the CONKO-04 approach of 'lifelong' thromboprophylaxis, the higher doses used throughout in our study compared to CONKO-04 (40 mg enoxaparin) do not allow direct comparisons. We demonstrate VTE control but note that thromboprophylaxis at late stages of advancing pancreatic cancer where bleeding becomes a more common event should be approached cautiously and the use of IDPTP beyond the 3 months used in the major studies should be reviewed on a case by case basis.

\section{Conclusion}

In conclusion we demonstrate that, an IDPTPLMWH with dalteparin reflecting the evidence can be implemented and patients can adhere to it. IDPTPLMWH was associated with lower ATTE occurrence rates than expected and comparable major bleeding rates. ATTE and MB were late events; the majority of $\mathrm{MB}$ was from the GI tract and the direct or indirect result of locally progressing aPDAC. Since these conditions can frequently arise in aPDAC, IDPTPLMWH beyond 3 months needs to be approached cautiously and should be regularly reviewed due to the increasing risk of GI bleeding in late -progressing- pancreatic cancer.

\section{Abbreviations \\ AG: Abraxane, Gemcitabine; aPDAC: Advanced Pancreatic Ductal Adenocarcinoma; ATTE: All Type Thromboembolic Events; BFI: Bleeding-free interval; DOAC: Direct Oral Anticoagulant; DVT: Deep Vein Thrombosis; ECOG-PS: Eastern Cooperative Group Performance status; FOLFIRINOX: Folinic Acid, 5-Fluorouracil, Irinotecan, Oxaliplatin; Gl: Gastrointestinal; GO: Gastro- oesophageal; IPE: Incidental Pulmonary Embolism; IDPTP: Increased Dose Primary Thromboprophylaxis; LMWH: Low Molecular Weight Heparin; MB: Major Bleeding; NNT: Numbers Needed to Treat; PE: Pulmonary Embolism; PEGPH20: Pegvorhyaluronidase Alfa; TE: Thromboembolic Event; TFI: TE-free Interval; VTE: Venous Thromboembolism}

\section{Acknowledgements}

We would like to thank Sarah Scargill for pharmacy crosschecking and Anita Gadie for support with the search of the HPB-MDT database.

\section{Authors' contributions}

AM Conceived the study, reviewed data, adjudicated/reconciled events and wrote the manuscript. FH Cleaned the data, analysed the data and reviewed the manuscript. IM developed the audit proposal, collected data, cleaned the data and reviewed the manuscript. WI Co-developed the audit proposal, collected data, developed the database, cleaned the data and reviewed the manuscript and submitted final audit report. GB Analysed the data, adjudicated/reconciled events and reviewed the manuscript. All Authors approved the final manuscript.

\section{Funding}

N/A

Availability of data and materials

Database of all 101 patients and statistical analyses available upon request and authorization from HUTH R\&D.

\section{Ethics approval and consent to participate}

Endorsement code for audit is 2017.98 issued by the Hull University Teaching Hospitals NHST approved the 1st June 2017. The study was performed in accordance with the data Protection Act (1998), the Caldicott principles (1997) and the NHS Confidentiality code of practice (2003) that all comply with the requirements of the declaration of Helsinki.

Consent for publication

N/A 


\section{Competing interests}

$\mathrm{FH}, \mathrm{IM}, \mathrm{WI}$ and $\mathrm{GB}$ have no conflicts of interest in relation to this work. AM has received fees for speaker's bureau, consultancy and travel from Pfizer (Dalteparin).

Received: 1 March 2020 Accepted: 11 May 2020

Published online: 29 May 2020

\section{References}

1. Maraveyas A. Pancreatic cancer: a model cancer for the study of the therapeutic effects of anticoagulants. World J Gastroenterol. 2009;1:38-40.

2. Khorana AA, Fine RL. Pancreatic cancer and thromboembolic disease. Lancet Oncol. 2004 Nov;5(11):655-63.

3. Menapace LA, Peterson DR, Berry A, Sousou T, Khorana AA. Symptomatic and incidental thromboembolism are both associated with mortality in pancreatic cancer. Thromb Haemost. 2011;106(2):371-8.

4. Lyman $\mathrm{GH}$, Eckert L, Wang $\mathrm{Y}$, Wang $\mathrm{H}$, Cohen A. Venous thromboembolism risk in patients with cancer receiving chemotherapy: a real-world analysis. Oncologist. 2013;18:1321-9. https://doi.org/10.1634/ theoncologist.2013-0226.

5. Khorana AA, Dalal MR, Lin J, Connolly GC. Health care costs associated with venous thromboembolism in selected high-risk ambulatory patients with solid tumors undergoing chemotherapy in the United States. Clinicoecon Outcomes Res. 2013;5:101-8.

6. Hidalgo M, Cascinu S, Kleeff J, Labianca R, Löhr JM, Neoptolemos J, et al. Addressing the challenges of pancreatic cancer: future directions for improving outcomes. Pancreatology. 2015;15(1):8-18.

7. Maraveyas A, Muazzam I, Noble S, Bozas G. Advances in managing and preventing thromboembolic disease in cancer patients. Curr Opin Support Palliat Care. 2017:11(4):347-54.

8. Maraveyas A, Waters J, Roy R, Fyfe D, Propper D, Lofts F, et al. Gemcitabine versus gemcitabine plus dalteparin thromboprophylaxis in pancreatic cancer. Eur J Cancer. 2012;48:1283-92.

9. Pelzer U, Opitz B, Deutschinoff G, Stauch M, Reitzig PC, Hahnfeld S, et al. Efficacy of prophylactic low-molecular weight heparin for ambulatory patients with advanced pancreatic cancer: outcomes from the CONKO-004 trial. J Clin Oncol. 2015;33:2028-34

10. Lyman GH, Khorana AA, Kuderer NM, Lee AY, Arcelus Jl, Balaban EP, et al. Venous thromboembolism prophylaxis and treatment in patients with cancer: American Society of Clinical Oncology clinical practice guideline update. J Clin Oncol. 2013;31:2189-204.

11. National Comprehensive Cancer Network Venous Thromboembolic Disease Clinical Practice Guidelines in Oncology. v.1.2015 edn. www.ncen.org: NCCN, 2015, Guideline.\#.

12. Farge D, Debourdeau P, Beckers M, Baglin C, Bauersachs RM, Brenner B, et al. International clinical practice guidelines for the treatment and prophylaxis of venous thromboembolism in patients with cancer. J Thromb Haemost. 2013;11(1):56-70.

13. Khorana AA, Soff GA, Kakkar AK, Vadhan-Raj S, Riess H, Wun T, et al. CASSINI investigators. Rivaroxaban for Thromboprophylaxis in high-risk ambulatory patients with cancer. N Engl J Med. 2019;380(8):720-8.

14. Carrier M, Abou-Nassar K, Mallick R, Tagalakis V, Shivakumar S, Schattner A, et al. Apixaban to prevent venous thromboembolism in patients with cancer. N Engl J Med. 2019;380(8):711-9.

15. Venous thromboembolism in over 16s: reducing the risk of hospital acquired deep vein thrombosis or pulmonary embolism: NICE guidline 21 March 2018. https://www.nice.org.uk/guidance/ng89.

16. Ek L, Gezelius E, Bergman B, Bendahl PO, Anderson H, Sundberg J, et al. Swedish lung cancer study group (SLUSG). Randomized phase III trial of low-molecular-weight heparin enoxaparin in addition to standard treatment in small-cell lung cancer: the RASTEN trial. Ann Oncol. 2018;29(2):398-404.

17. Schulman S, Kearon C. Subcommittee on control of anticoagulation of the scientific and standardization Committee of the International Society on thrombosis and Haemostasis definition of major bleeding in clinical investigations of antihemostatic medicinal products in non-surgical patients. J Thromb Haemost. 2005;3(4):692-4.

18. HRA. Defining research. NHS health Rresearch authority; 2017. http://www hra-decisiontools.org.uk/research/docs/DefiningResearchTable_Oct2017-1. pdf. Accessed 30 Dec 2017
19. HQIP. Best practice in clinical audit; 2016. Health quality improvement partnership. http://www.hqip.org.uk/resources/best-practice-in-clinical-audithqip-guide/. Accessed 30 Dec 2017.

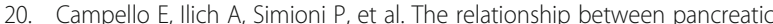
cancer and hypercoagulability: a comprehensive review on epidemiological and biological issues. Br J Cancer. 2019;121:359-71. https://doi.org/10.1038/ s41416-019-0510-x.

21. Conroy T, Desseigne F, Ychou M, Bouché O, Guimbaud R, Bécouarn Y, et al. FOLFIRINOX versus gemcitabine for metastatic pancreatic cancer. N Engl J Med. 2011;364:1817-25 10.1056.

22. Hingorani SR, Zheng L, Bullock AJ, Seery TE, Harris WP, Sigal DS, et al. HALO 202: randomized phase II study of PEGPH20 plus nab-paclitaxel/gemcitabine versus nab-paclitaxel/gemcitabine in patients with untreated, metastatic pancreatic ductal adenocarcinoma. J Clin Oncol. 2018;36(4):359-66.

23. Khorana AA, Dalal M, Lin J, Connolly GC. Incidence and predictors of venous thromboembolism (VTE) among ambulatory high-risk cancer patients undergoing chemotherapy in the United States. Cancer. 2013;119(3):648.

24. Wang YU, Yuan C, Liu X. Characteristics of gastrointestinal hemorrhage associated with pancreatic cancer: a retrospective review of 246 cases. Mol Clin Oncol. 2015;3(4):902-9.

25. Khorana AA, McCrae KR, Milentijevic D, Fortier J, Nelson WW. Laliberté F, et al current practice patterns and patient persistence with anticoagulant treatments for cancer-associated thrombosis. Res Pract Thromb Haemost. 2017;1(1):14-22.

26. Noble S, Matzdorff A, Maraveyas A, Holm MV, Pisa G. Assessing patients' anticoagulation preferences for the treatment of cancer-associated thrombosis using conjoint methodology. Haematologica. 2015;100(11): 1486-92.

27. Hutchinson A, Rees S, Young A, Maraveyas A, Date K, Johnson MJ. Oral anticoagulation is preferable to injected, but only if it is safe and effective: an interview study of patient and carer experience of oral and injected anticoagulant therapy for cancer-associated thrombosis in the select-d trial. Pal Med (Manuscript accepted for publication). 2019;33(5):510.

28. Yu KH, Mantha S, Tjan C, Kaufmann ES, Brenner R, Lowery MA. Pilot study of gemcitabine, nab-paclitaxel, PEGPH20, and rivaroxaban for advanced pancreatic adenocarcinoma: an interim analysis. J Clin Oncol. 2018;36(4_ suppl):405.

29. Young AM, Marshall A, Thirlwall J, Chapman O, Lokare A, Hill C, et al. Comparison of an Oral factor Xa inhibitor with low molecular weight heparin in patients with cancer with venous thromboembolism: results of a randomized trial (SELECT-D). J Clin Oncol. 2018;36(20):2017-23.

30. Raskob GE, van Es N, Verhamme P, Carrier M, Di Nisio M, Garcia D, et al. Edoxaban for the treatment of cancer-associated venous thromboembolism. N Engl J Med. 2018;378(7):615-24.

31. Agnelli G. Direct Oral anticoagulants for Thromboprophylaxis in ambulatory patients with cancer. N Engl J Med. 2019;380(8):781-3.

\section{Publisher's Note}

Springer Nature remains neutral with regard to jurisdictional claims in published maps and institutional affiliations.

Ready to submit your research? Choose BMC and benefit from

- fast, convenient online submission

- thorough peer review by experienced researchers in your field

- rapid publication on acceptance

- support for research data, including large and complex data types

- gold Open Access which fosters wider collaboration and increased citations

- maximum visibility for your research: over $100 \mathrm{M}$ website views per year

At $\mathrm{BMC}$, research is always in progress.

Learn more biomedcentral.com/submission 